\title{
ON ELECTRON ACCELERATION IN LIQUID RUPTURES CAUSED BY ELECTROSTRICTIVE FORCES
}

\author{
1'Petr BíLEK, 'Ján TUNGLI, 'Zdeněk BONAVENTURA \\ ${ }^{1}$ Department of Physical Electronics, Fac. Sci., Masaryk University, Brno, Czech Republic, EU, \\ petrbilek248@gmail.com
}

https://doi.org/10.37904/nanocon.2020.3728

\begin{abstract}
Water is often used as a medium for preparation of nanoparticles in plasma. One way to obtain plasma in liquids is a nanosecond high voltage pulse applied on micrometer sharp electrode, so that the electric discharge is developed. Liquid water under the action of sharp pulse of the electric field may be disrupted so that cavities of nanometer scale would eventually appear and expand. Owing the electric field forces, those nanocavities rapidly elongate to the form of long needle-like ruptures in the liquid bulk. We study electron acceleration in these ruptures and analyze the production of secondary electrons in the water near the nanocavity surfaces. For electron transport in the nanocavity and for electron water-interactions we use Monte Carlo model based on Geant4-DNA simulation toolkit. Nanocavities are modelled as hollow cylindrical voids in liquid water with homogeneous electric field inside oriented along the cylindrical axis. Due to the nanometric scale of these voids, electrons can move collisionless inside, where are also accelerated by the action of the electric field. Primary electrons are injected as monoenergetic isotropic source from the inner surface of the void. We seek physical conditions, a combination of electric field strength and geometry of the cavity that would lead to the production of more than one secondary electron per single primary electron. This study is relevant for understanding of initial phases of electric discharge development in liquid water.
\end{abstract}

Keywords: In-liquid plasma, nanocavities, electrostriction, electron multiplication

\section{INTRODUCTION}

Applications of plasma in many distinct technological areas has increased gradually, ever since the term 'plasma' has been coined by an American chemist and Nobel Prize winner Irving Langmuir in 1920s. Historically, plasma has been used as a medium to generate chemically active species in various gas mixtures. During past few decades, attempts to generate plasma also in liquid environment has emerged, followed immediately by applications such as nanomaterials syntheses and wastewater treatment $[1,2]$. Many of the currently reported devices for plasma generation in liquids use presence of diluted bubbles in the liquid, where accelerated electrons ionize vapors of the liquid. Then, the electric breakdown, in fact, takes place in gaseous environment instead of directly in the liquid bulk. Typical voltage pulses used for the generation of such plasmas are of microsecond duration, applied to a sharp metallic electrode, e.g. in point-plane electrode geometry [3]. Another interesting case to consider for plasma generation in liquids is an ultra sharp highvoltage pulse of (sub)nanosecond duration. Currently, the prevailing opinion is that, in that case, for polar liquids such as water, the initial stage of the plasma discharge formation is associated with the appearance of nanoruptures or nanovoids, which occur because of the ponderomotive electrostrictive forces. Inside of these nanovoids, then the electrons might gain sufficient energy to ionize. Due to the action of the electric forces, these nanocavities rapidly elongate and form long needle-like ruptures of the liquid bulk $[4,5]$. Note that this mechanism of plasma generation is different from the bubble-electric-breakdown, because nanovoids serve as collision-free environment merely for acceleration of electrons and ionization occurs in the liquid, instead. 
In this contribution, we study electron acceleration in these elongated ruptures and we analyze resulting production of secondary electrons in the water near the nanocavity/water interface.

\section{MODEL AND METHODS}

Cavities are considered as long cylindrical voids with homogeneous electric field loaded inside and oriented along their axis. Electrons in the cavity accelerate freely, i.e. without collisions, and can only interact with water molecules when they hit the wall of the cavity. This assumption of uncolissionality is justified because the characteristic spatial scale of these cavities is much shorter than the mean free path of electrons in the corresponding equilibrium vapor pressure. The electron motion in the cavity with the homogenous electric field is an analogy to the projectile motion in a vertical tube subjected to the action of the gravitation force. It can be shown that the product of the electric field strength $E$ and the cavity radius $R$, i.e., $E \cdot R$ is appropriate for discussion of results for different cavity dimensions and electric field strengths. For description of electron interactions with water, we use state of the art simulation framework Geant4-DNA [6-9]. This framework offers a variety of models to simulate the physical interactions of electrons in liquid water. We use Geant4-DNA physics 'option 4' constructor, which includes models for elastic and inelastic scattering of electrons, together with a model for vibrational excitation and attachment. We assume that sub-excitation electrons, i.e. electrons with energy below $7.4 \mathrm{eV}$, cannot contribute to further ionization in water. Therefore, no tracking of subexcitation electrons in water is performed in the simulation. Overall, the electron interaction with the surface of the cavity can have two distinct outcomes: (a) the electron penetrates the water bulk and terminates there, or (b) the electron is bounced back to the void of the cavity and is accelerated by the electric field once again. In both cases, in termination or in bouncing, certain number of secondary electrons is created in the water by electron impact ionization of water molecules. In addition, some portion of those secondary electrons is emitted from the surface of the cavity to the void. In the following, we analyze this production of secondary electrons in detail, and we look for suitable condition to ensure that the number of secondary electrons emitted to the cavity per one impacting primary electron would be equal to one or higher. Primary electrons are launched from the surface of the cavity with isotropic velocity distribution and the initial energy at the lowest threshold energy $7.4 \mathrm{eV}$. This initial condition is motivated by three facts. First, every secondary electron can serve as a primary electron for the next generation of secondary electrons. Second, electron collisionality in the water is very high so that any electron that is emitted or bounced-back from the cavity/water interface to the cavity will have no memory of momentum prior to the latest collisional event. Therefore, we can assume isotropic initial velocity distribution of primary electrons. And third, any electron with sub-excitation energy will thermalize in the water bulk and cannot further produce secondary electrons. Minimal energy that allows electrons to be released to the cavity is therefore at the threshold of $7.4 \mathrm{eV}$ in our model. Then the initial condition formulated this way allows us to study the production of secondary electrons and make some conclusions regarding the conditions that are favorable for electron multiplication inside the nanocavity.

\section{RESULTS}

Figure 1 shows distribution of secondary electrons generated along the cavity in water by the impact of primary electrons released from the cavity surface at position $z=0$. The distribution is normalized such a way that the integral of all secondary electrons emitted in the cavity is divided by the total number, $\mathrm{N} \_0$, of primary electrons launched. Panels on Figure 1 represent secondary electron distribution for $E \cdot R$ in $\{15,18,20,25\} V$. Total number of secondaries per single primary electrons are $\{0.75,1.01,1.15,1.5\}$, this number is obtained as a sum of all secondary electrons generated and emitted along the cavity. In this regard it is interesting to look at the cumulative sum of secondary electrons along the cavity length, as shown in Figure 2 for $E \cdot R$ in $\{15,18,20,25\} V$. Clearly for $E \cdot R$ higher or equal to 18 , there is a finite length of the tube on which the number of secondary electrons per single primary electron sums up to one or higher number. On the other hand for $E$ $\cdot R=15 \mathrm{~V}$, there will be less secondary electrons then primary electrons, no matter how long the cavity would 
be. This allows us to conclude that there exists a condition on a minimal value of $E \cdot R$ that is required for electrons released into the cavity to be multiplied by ionization along the cavity surface.
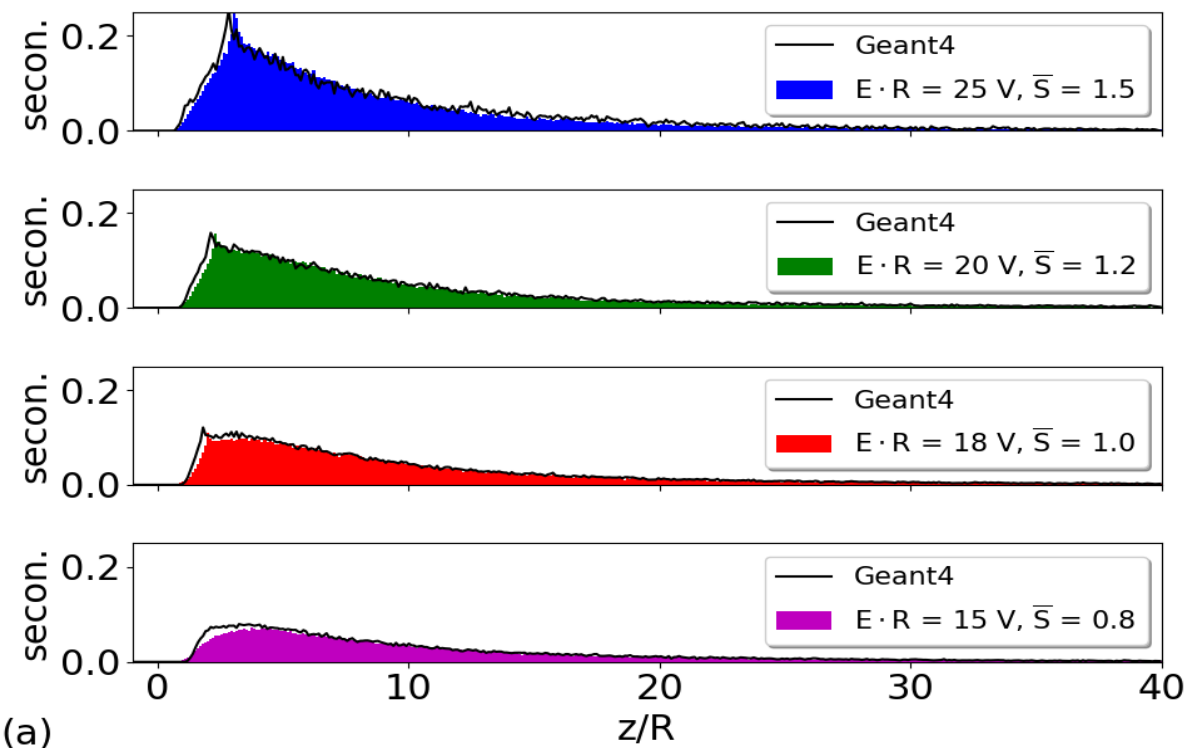

Figure 1 Distribution functions of secondary electrons along $z$-axis for four different $E \cdot R$ values. Integrals of the distribution functions along z-axis give number of secondary electrons generated per primary electron

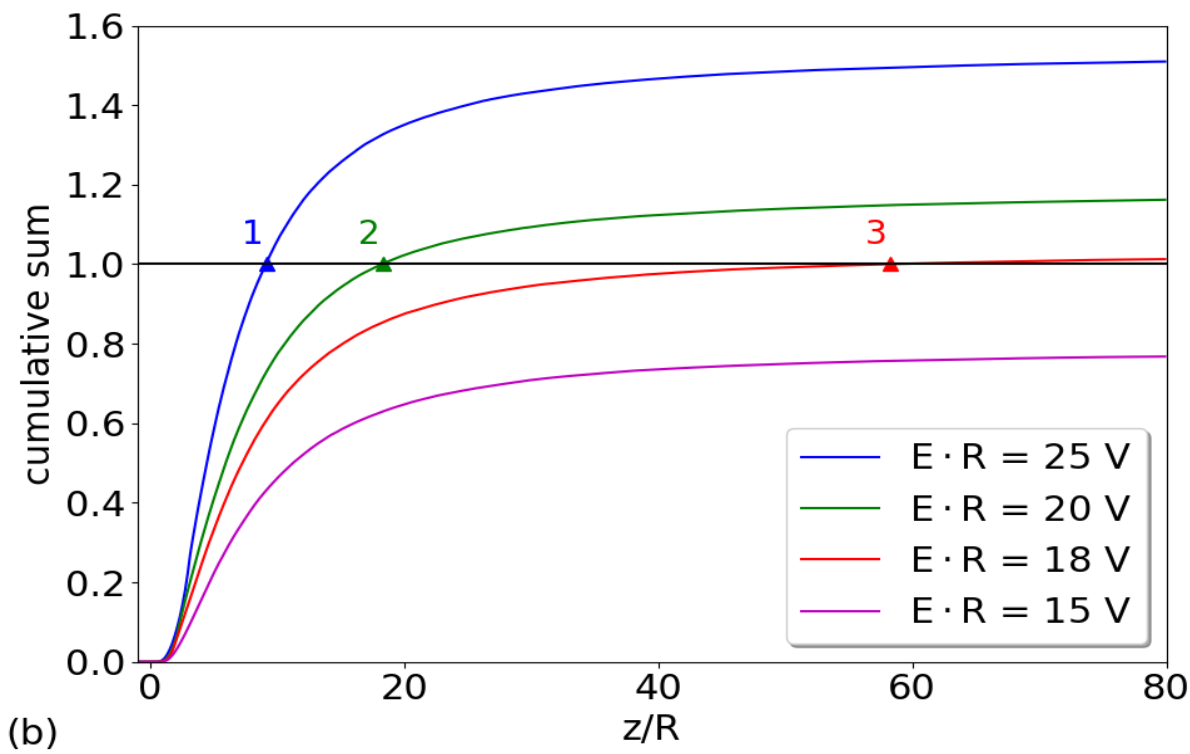

Figure 2 Cumulative sums of the distribution functions and the dots denote the length of the cavity for which the primary electron produces exactly one secondary electron

\section{CONCLUSION}

We have simulated motion of primary electrons released in the capillary rupture created by electrostrictive stress in water by strong and ultrafast pulse of the electric field. Electrons are accelerated by the electric field in the void of the rupture under collisionless conditions. Then by impact on the surface of the cavity, we calculate number of secondary electrons emitted from the cavity surface by these accelerated impacting 
primary electrons. Considering several values of electric field strength times cavity radius $(E \cdot R)$, we observe that there exists a minimal value of $E \cdot R(\sim 18 \mathrm{~V})$ at which the number of primary electrons is ensured to be multiplied. These results has strong implications for understanding of initial phases of electric discharge and plasma state development in liquid water.

\section{ACKNOWLEDGEMENTS}

\section{This work was supported by the Czech Science Foundation (Project ID 18-04676S).}

\section{REFERENCES}

[1] HORIKOSHI, S., SERPONE, N. In-liquid plasma: a novel tool in the fabrication of nanomaterials and in the treatment of wastewaters. RSC Advances. 2017, vol. 7, pp. 47196-47218.

[2] SAITO, G., AKIYAMA, T. Nanomaterial synthesis using plasma generation in liquid. J. Nanomater. 2015, pp. 121.

[3] DEVINS, J.C., RZAD, S.J., SCHWABE, R.J. Breakdown and pre-breakdown phenomena in liquids. J. Appl. Phys. 1981, vol. 52, pp. 4531-4545.

[4] SHNEIDER, M.N., PEKKER, M. Pre-breakdown processes in a dielectric fluid in inhomogeneous pulsed electric fields. Journal of Applied Physics. 2015, vol. 117, 224902.

[5] M.ŠIMEK et al. Investigation of the initial phases of nanosecond discharges in liquid water. Plasma Sources Sci. Technol. 2020, vol. 29, 064001.

[6] INCERTI, S., KYRIAKOU, I., BERNAL, M., BORDAGE, M., FRANCIS, Z., GUATELLI, S., IVANCHENKO, V., KARAMITROS, M. LAMPE, N., LEE, S. B., et al. Geant4-DNA example applications for track structure simulations in liquid water: A report from the Geant4-DNA Project. Medical physics. 2018, vol. 45, pp. e722-e739.

[7] BERNAL, M., BORDAGE, M., BROWN, J., DAVÍDKOVÁ, M., DELAGE, E., EL BITAR, Z., ENGER, S., FRANCIS, Z., GUATELLI, S., IVANCHENKO, V., et al. Track structure modeling in liquid water: A review of the Geant4-DNA very low energy extension of the Geant4 Monte Carlo simulation toolkit, Physica Medica. 2015, vol. 31, pp. 861874.

[8] INCERTI, S., IVANCHENKO, A., KARAMITROS, M., MANTERO, A., MORETTO, P., TRAN, MASCIALINO, B., CHAMPION, C. IVANCHENKO, V., BERNAL, M., et al. Comparison of GEANT4 very low energy cross section models with experimental data in water. Medical physics. 2010, vol. 37, pp. 4692-708.

[9] INCERTI, S., BALDACCHINO, G., BERNAL, M., CAPRA, CHAMPION, C., FRANCIS, Z., GUATELLI, S., GUĖYE, P., MANTERO, A., MASCIALINO, B., MORETTO, P., NIEMINEN, P., ROSENFELD, A., VILLAGRASA C., ZACHARATOU, C. THE GEANT4-DNA PROJECT. Int. J. Model. Simul. Sci. Comput. 2010, vol. 1, pp. 157--178. 